\title{
MICROMIRROR PIXEL ADDRESSING USING ELECTROMECHANICAL BISTABILITY
}

\author{
Brian T. Cunningham, Jonathan J. Bernstein, Don Seltzer, and David Hom \\ The Charles Stark Draper Laboratory \\ 555 Technology Square, Cambridge, MA 02139
}

\begin{abstract}
In this work, a novel bistable array of micromirrors (BAMM) has been designed, fabricated, and tested which utilizes the electromechanical properties of the flexural hinged mirror elements to store the desired individual mirror states. Using the mirror structures as electromechanically bistable storage devices, pixels can be addressed individually without the use of integrated static random access memory (SRAM) cells beneath each element. Through the elimination of integrated RAM circuitry, the mirror array fabrication sequence is greatly simplified, and decreased yielded die costs are expected compared to fabrication which relies upon the combined yield of the memory and mirror processes.
\end{abstract}

\section{INTRODUCTION}

The commercialization of MEMS spatial light modulator (SLM) arrays has been pioneered by Texas Instruments and others through the development of the Digital Micromirror Device (DMD) and deformable grating light valve arrays for use in digital high resolution color projection displays [1-4]. The DMD pixel elements consist of reflective aluminum platforms which can be rotated about a torsion hinge into one of two bistable states using electrostatic electrodes beneath the platform. The MEMS structure is typically fabricated monolithically over a CMOS SRAM cell which is used to address the pixel by digitally storing the desired state of the mirror for the subsequent video frame.

While integration of the CMOS process with the MEMS process may be the most economically advantageous fabrication method for applications where high sales volume and large capital equipment resources permit, many applications with smaller expected markets require arrays of SLMs which can be individually addressed. For such applications, simplification of the fabrication process and the ability to utilize commercially available electronic components is key to providing SLM arrays with low cost and high yield. The impact of fabrication yield is especially important for video display applications where a defect within a single pixel during the SRAM or MEMS portion of the process will result in rejection of an entire large area array.

In this work, a bistable array of micromirrors (BAMM) is presented which enables individual pixel row/column addressing without the use of an SRAM cell beneath each pixel. The addressing method allows one to set the desired state of every mirror in a selected row simultaneously while holding all other rows fixed. The on/off state of every pixel in the array is set by addressing the rows in sequence. $10 \times 10$ and 100×100 mirror arrays were fabricated using the MCNC MUMPs process to demonstrate the BAMM addressing approach. In principle, the BAMM addressing method is applicable to bistable mirror arrays fabricated by any process. Using the BAMM addressing approach, mirror arrays can be fabricated with only 6 photomask steps, eliminating the need for a special CMOS SRAM wafer and processes associated with interfacing CMOS with MEMS such as surface planarization and interconnect vias. Through the use of a stand-alone MEMS process, high temperature materials such as polysilicon can be considered which would otherwise not be compatible with finished CMOS circuitry. The use of polysilicon hinges may result in longer pixel lifetime compared to aluminum hinges which can show plastic and fatigue behavior. A simple, inexpensive switch relay network was designed and built to operate the 10x10 array which can easily be scaled to address larger arrays.

\section{THEORY}

A schematic drawing of a BAMM pixel element is shown in Figure 1. The reflective platform pivots around a thin torsion hinge when a voltage applied to either the "left" or "right" electrode breaks the symmetry while an electrostatic snapdown voltage is applied with the "hold" electrode. Once the platform is deflected to its desired state, the "hold" electrode maintains the pixel's position until it is released for a subsequent write cycle. Using this method, a row of pixels in an $x-y$ array can be addressed by writing to one row while all other rows are "held" as shown in Figure 2. A timing sequence for the addressing process is shown in Figure 3 .

The rotational snapdown voltage of the pixel is given by

$$
v_{S D}=\sqrt{\frac{k_{\theta} x_{\theta}^{3}}{2 \varepsilon A_{c} L_{c}^{2}}}
$$

where $k_{\theta}$ is the rotational spring constant of the torsion hinge $(\mathrm{N}-\mathrm{m} / \mathrm{rad}), x_{0}$ is the quiescent capacitor gap, $\varepsilon$ is the dielectric constant of air, $A_{c}$ is the left or right side area, and $L_{c}$ is the distance from the center line to the center of the electrode. The design goal, within the constraints of the MUMPs fabrication process design rules, was to produce pixels with $\mathrm{V}_{\mathrm{SD}}<15 \mathrm{~V}$. Because $\mathrm{x}_{(1)}$ is fixed by the MUMPs process at $x_{0}$ $\sim 2 \mu \mathrm{m}$, devices were fabricated with various pixel sizes to affect $A_{c}$ and $L_{c}$ and various flexure widths to affect $k_{\theta}$.

\section{EXPERIMENTAL RESULTS}

For demonstration of the BAMM concept. $50 \times 50 \mu \mathrm{m}$ and $100 \times 100 \mu \mathrm{m}$ pixels were designed with flexure widths of $1.0,1.5$, and $2.0 \mu \mathrm{m}$. $1.0 \mu \mathrm{m}$ was the smallest linewidth allowed by MUMPs. Two types of pixels were fabricated: exposed flexures and hidden flexures. While both types of pixels use flexures and a rotational platform built from the 2.0 $\mu \mathrm{m}$ thick Poly 1 layer, the hidden flexure designs incorporate an additional upper metalized ( $\mathrm{Cr} / \mathrm{Au}) 1.5 \mu \mathrm{m}$ Poly 3 layer to increase optical fill factor and to provide a more reflective top surface. A $100 \times 100 \mu \mathrm{m}$ pixel element with a $1 \mu \mathrm{m}$ wide hidden hinge is shown in Figure 4. Selected pixel designs were incorporated into $10 \times 10$ and $100 \times 100$ element arrays. A 
portion of a $100 \times 100$ element array with $1.5 \mu \mathrm{m}$ exposed flexures is shown in Figure 5.

Pixel snapdown voltage was characterized for the various designs and flexure widths. As shown in Figure 6, $50 \times 50 \mu \mathrm{m}$ pixels were found to require excessive $V_{S D}$ for all flexure widths while $100 \times 100 \mu \mathrm{m}$ pixels using the hidden flexure design with $1.0 \mu \mathrm{m}$ wide flexures required a $\mathrm{V}_{\mathrm{SD}}$ of only $9 \mathrm{~V}$. Due to chip area constraints, $10 \times 10$ element arrays with the hidden flexure design were only fabricated using 1.5 $\mu \mathrm{m}$ wide flexures, with $\mathrm{V}_{\mathrm{SD}}=18 \mathrm{~V}$. Therefore, demonstration of the BAMM addressing approach was performed with this design. As shown in Figure 6, device-to-device snapdown voltage repeatability was excellent.

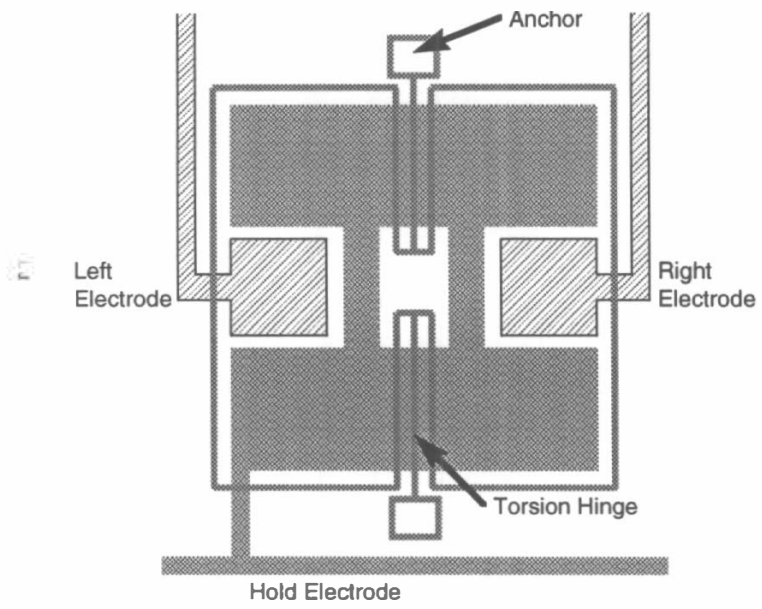

Figure 1. Schematic drawing of a BAMM pixel element. The mirror platform is supported by a central thin torsion hinge. The "Left" and "Right" electrodes are used to break the symmetry when an electrostatic snapdown voltage is applied with the "Hold" electrode. The "Hold" electrode extends across the center of the pixel to keep the pixel in its intended state until it is released for the next write cycle.

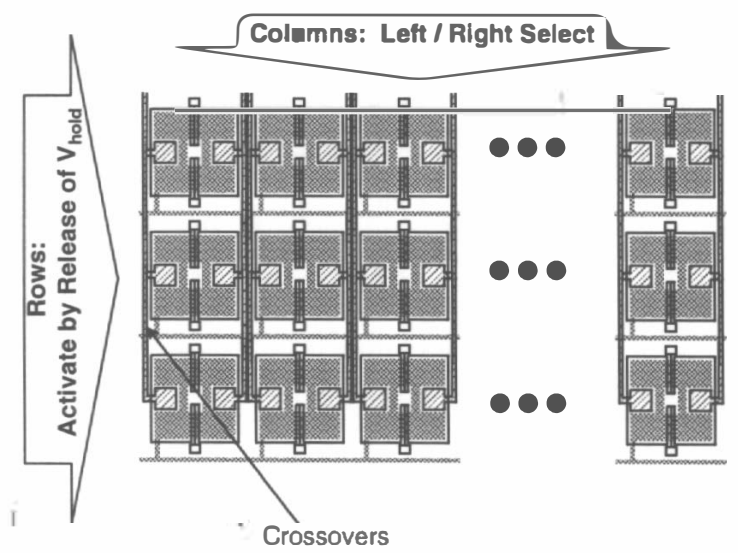

Figure 2. The left/right state is selected for every column simultaneously. By releasing the "Hold" voltage from a single row, only one row is affected by the selected left/right state. Array pixel addressing is achieved by sequentially releasing $V_{\text {hold }}$ for each row, synchronized with the application of desired left/right state from the column lines.

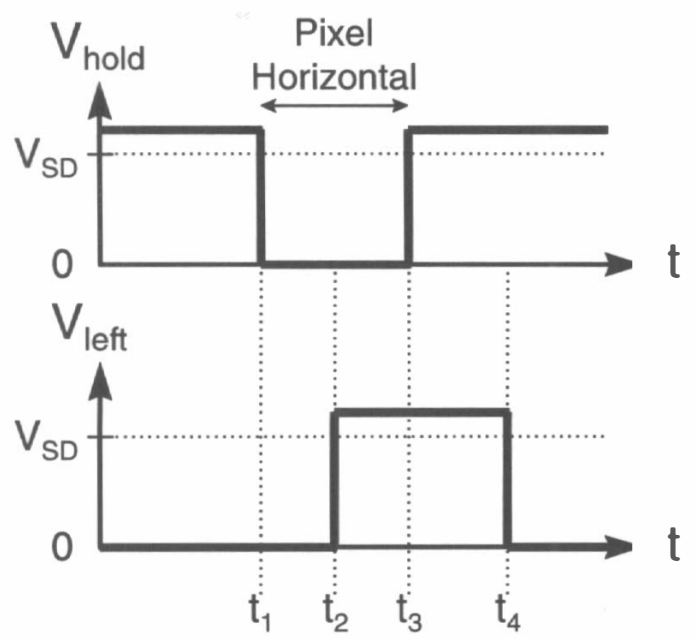

Figure 3. Example tuming sequence for directing $a$ pixel to rotate to its "left" state. After $V_{\text {hold }}$ is released for a row at $t_{1}$, either $V_{\text {left }}$ or $V_{\text {right }}$ is activated at $t_{2}$ to direct the pixel. When $V_{\text {hold }}$ is reapplied, the pixel snaps to the desired state, and $V_{\text {left }}$ is reset at $t_{4}$ to address the state of the next row. The pixel is up for $t_{1}<t<t_{3}$ while $V_{\text {hold }}$ is released.

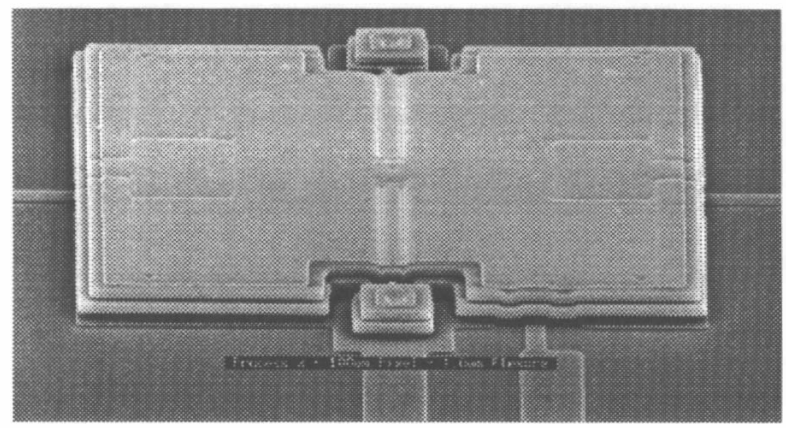

Figure 4. SEM photo of $100 x 100 \mu \mathrm{m}$ pixel with $1.0 \mu \mathrm{m}$ wide hidden flexure.

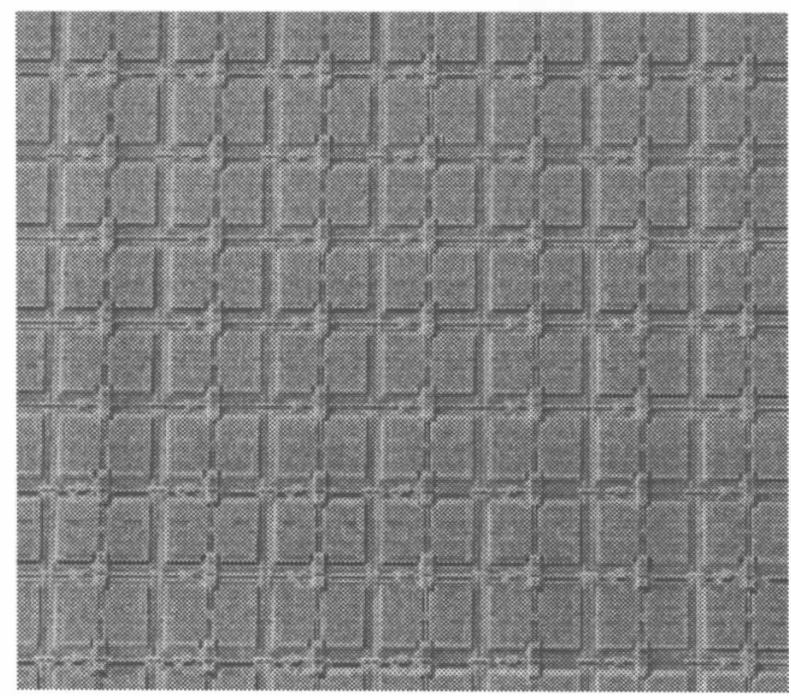

Figure 5. SEM photo of portion of a $100 \times 100$ element BAMM array with 100x100 $\mathrm{m}$ pixels and $1.5 \mu \mathrm{m}$ wide exposed flexures. 


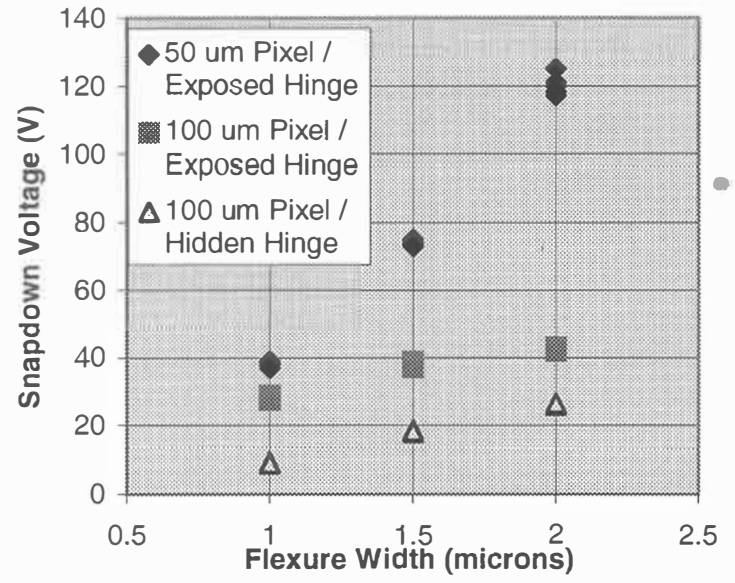

Figure 6. Snapdown voltage as a function of flexure width for three BAMM pixel designs. Five pixels are sampled for each design and flexure width.

Mirror control electronics were built to demonstrate functionality of the $10 \times 10$ element hidden $1.5 \mu \mathrm{m}$ flexure array. Because $\mathrm{V}_{\mathrm{SD}}=18 \mathrm{~V}$ for this design, high voltage components were required for switching the "hold" and "leftright" select electrodes. As shown in Figure 7, a computer is used to control the high voltage relay network which sequentially writes column data to rows which are activated by removal of the "hold" voltage. Using computer control, sequences of programmed pixel patterns can be displayed by the mirror array. For this work, equal voltages were applied to the "hold" and "left/right" electrodes. For pixels with a measured $\mathrm{V}_{\mathrm{SD}}=18 \mathrm{~V}$, an applied voltage of $\mathrm{V}=$ $20 \mathrm{~V}$ was found to give the most reliable operation. Operation with $\mathrm{V}>30 \mathrm{~V}$ was found to result in a small percentage of "sticky" pixels which would remain fixed in one state for several cycles, but which would eventually release. No effort was made to apply anti-stiction coatings, although the pixels incorporate small dimples on the bottom sides of the mirror elements to minimize surface contact area during snapdown.

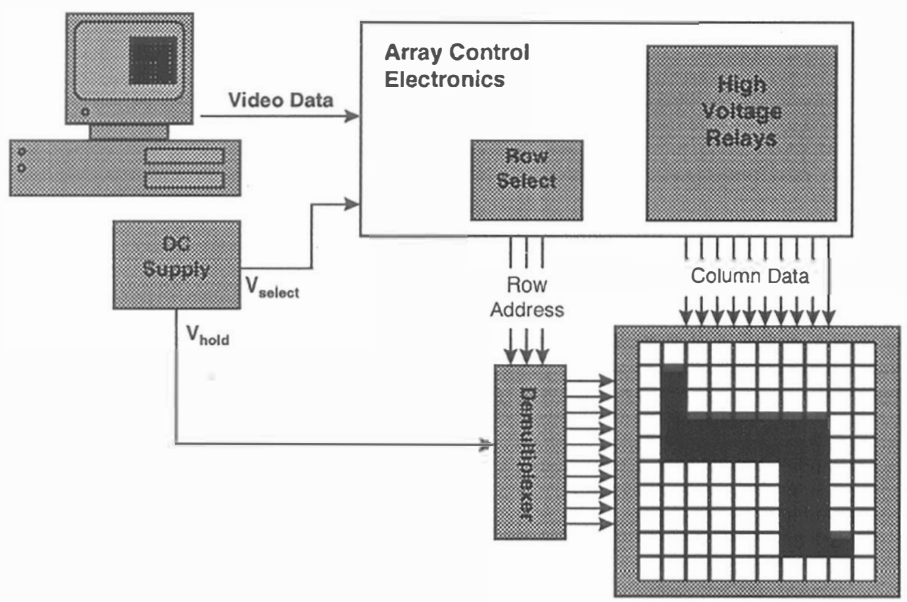

Figure 7. Mirror array control electronics for operation of a $10 x 10$ element BAMM array.

\section{CONCLUSION}

A bistable mirror array addressing approach has been demonstrated which enables individual pixel addressing without the use of an integrated SRAM memory cell. The approach relies upon the electromechanical bistability of the mirror pixel's torsional hinge to maintain the position of the array elements while a single row is addressed. The method was demonstrated using $10 \times 10$ polysilicon mirror arrays fabricated with the MCNC MUMPs process. The BAMM approach is broadly applicable to SLM arrays of mirrors or deformable gratings fabricated by any method.

Further optimization work would focus on using chemical-mechanical polishing (CMP) to provide a smoother top surface, as well as addition of an upper aluminum metalization layer for increased reflectivity. The use of thinner and narrower flexures will allow reduction of the applied voltages to $<5 \mathrm{~V}$ for compatibility with standard CMOS electronics.

\section{ACKNOWLEDGEMENTS}

The authors would like to acknowledge the Draper Laboratory MEMS Center of Excellence for providing the funding for this project, and to Dr. Marc Weinberg and Dr. Byong Ahn for their management interest and support.

\section{REFERENCES}

1. L. Hornbeck, Proc. of Micromachining and Microfabrication '95, Plenary Session, Austin, TX, October 23-24.

2. V.P. Jaecklin, Sensors and Actuators A, 43, p. 269 (1994).

3. E. Chiu, 1995 Intl. Symp. On VLSI Technology, Systems, and Applications, p. 137.

4. R. Apte, Technical Digest of the 1994 Sensor and Actuator Workshop, Hilton Head Isl., SC, p 1-6. 